\title{
ALGUNAS POTENCIALIDADES EPISTÉMICAS DE LA PEDAGOGÍA CRÍTICA
}

\author{
Carlos Arturo Muñoz Vargas ${ }^{1}$ \\ María Isabel Afanador Rodríguez ${ }^{2}$
}

Resumen: El artículo corresponde a un producto del proyecto de investigación: Concepción pedagógica para la formación en Pedagógica Crítica de los profesores de matemáticas de la Institución Universitaria Antonio José Camacho, de Cali, Colombia; adscrito a Grupo de Investigación en Pedagogía GIP-UNIAJC; el cual tiene el propósito de realizar un acercamiento a las potencialidades epistémicas de la Pedagogía Crítica para la comprensión teóricometodológica de la práctica educativa del profesor universitario.

Palabras clave: Epistemología, conocimiento, pedagogía crítica, sociedad, política.

\section{ALGUMAS POTENCIALIDAdES EPISTÉMICAS DA PEDAGOGIA CRÍTICA}

Resumo: O artigo corresponde a um produto do projeto de pesquisa: Concepção Pedagógica para a Formação Pedagógica Crítica dos Professores de Matemática da Universidade de Antonio José Camacho, de Cali, Colômbia; atribuída ao Grupo de Pesquisa em Pedagogia do GIP-UNIAJC; que visa abordar o potencial epistêmico da Pedagogia Crítica para a compreensão teórico-metodológica da prática educativa do professor universitário.

Palavras-chave: Epistemologia, conhecimento, pedagogia crítica, sociedade, política.

\section{SOME EPISTEMIC POTENTIALITIES OF CRITICAL PEDAGOGY}

\begin{abstract}
The article corresponds to a product of the research project: Pedagogical Conception for the Critical Pedagogical Training of the Mathematics Professors of the Antonio José Camacho University Institution, of Cali, Colombia; ascribed to the Research Group in Pedagogy GIP-UNIAJC; which aims to make an approach to the epistemic potential of Critical Pedagogy for the theoretical-methodological understanding of the educational practice of the university professor.
\end{abstract}

Keywords: Epistemology, knowledge, critical pedagogy, society, policy.

\section{Introducción:}

A partir de sus propias intenciones, este trabajo pretender hacer un acercamiento a las potencialidades epistémicas de la Pedagogía Crítica para la comprensión teórico-metodológica de la

\footnotetext{
${ }^{1}$ Investigador del Grupo de Investigación en Pedagogía GIP-UNIAJC, docente nombrado de carrera, Asociado de tiempo completo de la Institución Universitaria Antonio José Camacho, UNIAJC. Cali, Colombia. Director del Departamento de Ciencias Básicas de la UNIAJC. Ingeniero Electricista -Universidad Autónoma de OccidenteEspecialista en Gerencia de Instituciones Educativas -Universidad del Tolima, Magister en Educación Énfasis Educación Matemáticas de la Universidad del Valle y candidato a Doctor en Ciencias Pedagógicas de la Universidad en Ciencias Pedagógicas Enrique José Varona de La Habana, Cuba.email: cmunoz@admon.uniajc.edu.co; mecaito7@ hotmail.com

2 Investigadora del Grupo de Investigación en Pedagogía GIP-UNIAJC, docente de Carrera de la Institución Universitaria Antonio José Camacho, UNIAJC. Cali, Colombia. Decana de la Facultad de Educación a Distancia y Virtual de la UNIAJC. Licenciada en Educación Preescolar de la Universidad de San Buenaventura. Especialista en Gerencia Estratégica de Instituciones Educativas de la Universidad Autónoma de Occidente. Maestría en Tecnología Educativa y Medios Innovadores para la Educación de la Universidad Tecnológica de Monterrey y estudiante de Doctorado en Ciencias Pedagógicas de la Universidad en Ciencias Pedagógicas Enrique José Varona de La Habana, Cuba. Email: mafanador@admon.uniajc.edu.co; maria.isabel.afanador@gmail.com
} 
práctica educativa del profesor universitario, pasa ello necesariamente por la identificación de su estatus científico, máxime, hoy día, cuando en la literatura pedagógica existe una proliferación de términos afines: pedagogía de la diversidad, pedagogía de la humildad, pedagogía del amor, pedagogía comunitaria, pedagogía social, por citar algunos de ellos.

La discusión se da en el marco del eje relacional: educación/pedagogía crítica/sociedad, donde se destaca los agentes que intervienen en el acto pedagógico, estudiantes, profesores, institución; y los destinatarios sociales.

Según el criterio estructurador de los campos de acción de Bourdieu (1991, p. 27), en la Pedagogía crítica se reconocen los siguientes:

El campo científico: reconoce a los saberes y conocimientos que desde la Pedagogía Crítica se producen, o dicho de otro modo, su aportación epistemológica, teórica y metodológica en el campo de la educación social en las distintas universidades.

El campo disciplinar: reconoce el devenir de la Pedagogía crítica como materia académica incorporada a los planes de estudio de las licenciaturas en Pedagogía y Educación social, y Trabajo Social en las universidades.

El campo profesional: hace referencia a la práctica de los profesionales educadores- como actores inmersos en la cotidianidad de las prácticas en las instituciones educativas.

En esta dirección vale destacar que, según este criterio organizador, los campos analizados se nuclean alrededor de tal eje relacional: educación/pedagogía crítica/sociedad, cada uno de ellos remite a discursos, contextos y prácticas que marcan la acción más o menos institucionalizada de unos agentes profesionales y unos destinatarios sociales. Vista así, la Pedagogía Crítica se concibe como episteme, disciplina (materia de estudio) y práctica socioeducativa. Por tales razones, es necesario precisar cuál es el criterio a seguir en la estructura del conocimiento pedagógico crítico y así poder determinar los elementos esenciales de mayor afinidad con el objeto que se estudia.

\section{Desarrollo:}

La cientificidad de la Pedagogía Crítica no está en preguntarse por el objeto del conocimiento en sí mismo, es decir por qué se conoce, sino que la cuestión de mayor significación está en centrarse más cómo se construye el conocimiento pedagógico crítico, hace falta clarificar quiénes son los que están por detrás de ese conocimiento, cómo lo producen, de qué forma lo utilizan, qué valor le dan, qué uso, cómo lo recrean en su formación, a qué fines sirve.

Ante estas premisas expuestas, conviene realizar un acercamiento a lo que se concibe como Pedagogía Crítica y sus intereses. Tal epistemología, tomando como referente el concepto marxista 
de la reproducción social, se esfuerza por interpretar a la pedagogía desde sus interrelaciones con el mantenimiento y conservación de las relaciones sociales y políticas que imponen los sectores dominantes. De la educación le preocupa que, por tener siempre intereses sociales detrás de ella, se convierte en un instrumento de dominación o de emancipación. El debate tiene un sentido atractivo cuando se concibe a la Pedagogía Crítica como una "crítica a la pedagogía", este juego de palabras, interesante a criterio de los autores, determina que cualquier corriente de pensamiento crítico es sin duda un metalenguaje, es decir, es la Pedagogía Crítica un sistema de pensamiento, una episteme, que se crea y se recrea a partir de sus propias reflexiones sobre los significados conceptuales.

Del discurso pedagógico de la educación, es de mucha importancia que autores como Berstein (1998) la sitúen como una forma especializada de comunicación (Fonseca, 2011), pues se apropia de otros discursos para ponerlos en la escena educativa, de tal forma que construye socialmente el propio en las interacciones complejas entre los agentes en contexto. Desde este punto de vista, se llama la atención a la naturaleza de la práctica educativa, fundamentalmente protagonizada por el profesor, pues en ella convergen, además de otros discursos, imaginarios, códigos, dispositivos, estructuras, intereses, controles, pasiones, todos ellos condicionantes, sociales, humanos y técnicos que complejizan el proceso pedagógico y por tanto su estudio, producción y reproducción.

En efecto, así el discurso pedagógico se configura como un instrumento de poder en manos del profesor, que a través de la práctica consciente de los agentes del acto educativo será transformado o deformado; sea cual fuere el caso, el acto y el discurso pedagógico es proclive al amoldamiento (domesticación) de la conciencia y, por ello, a su ubicación funcional en términos definidos, con mayor o menor detalle, por parte del poder dominante (Fonseca, 2011). Por lo anterior, el acto educativo y, más el discurso pedagógico, debe ser fundamentalmente consciente; ello implica que el profesor, como agente educador, debe, además, tener la certeza y el compromiso que su práctica es transformadora, liberadora y emancipadora social.

En este orden de supuestos y premisas fundacionales de la razón de ser de la Pedagogía Crítica se encuentra una cuestión filosófica relacionada con la crítica al naturalismo de Rousseau sobre la naturaleza de la libertad humana, cuando se asume la premisa que las mujeres y los hombres no son por naturaleza libres, que habitan un mundo en constante cambio y que se debaten entre las contradicciones dialécticas de poder y represión, esto implica que el conocimiento o saber, como construcción social e histórica, adquirido o logrado en cualquier tipo de institución educativa, llámese escuela, universidad, obedece a intereses sociales definidos y, en consecuencia, nunca es 
neutral, sino que está diseñado, estructurado e implementado en forma particular con sus prioridades y exclusiones que hacen parte de una lógica soterrada por las clases de poder dominante (Muñoz, 2016).

Si bien no interesa aquí reducir la Pedagogía Crítica a un concepto, es significativo subrayar algunos principios rectores que la determinan (López, 2010): La relación teoría-práctica en cuanto a la generación social del conocimiento; la racionalidad crítica dialéctica como grado de autonomía del profesor en su proceso de autoliberación; la contextualización por el reconocimiento de la íntima relación al espacio-tiempo; la investigación acción deliberativa colaborativa como proceso situado colectivo y transformador de la práctica, la ética como valor intrínseco del sujeto sociopolítico.

La Pedagogía Crítica nace de las corrientes de pensamiento neomarxistas de la Teoría Crítica (1923), más tarde Escuela de Frankfurt (1950) que abordó estudios multidisciplinares en economía, política, filosofía, sociología y ética. Se reconocen tres generaciones de Pensamiento Crítico: la primera, a través de los postulados de autores de la altura de Horkheimer (1895-1973), Fromm (1900-1980), Adorno (1903-1969) y Marcuse (1898-1979) que se enfocaron en la reflexión ética crítica al dominio de la civilización de la postguerra; más adelante la segunda, donde se destaca Habermas (1929- ) con su teoría de la acción comunicativa; y la tercera, con autores como Touraine, Guides y Dubiel, donde cuestionan el debilitamiento de las economías de los estados nacionales frente al surgimiento de las corporaciones. Sin embargo, autores como Morales (2014) consideran que las ideas de la Pedagogía Crítica no se extraen de los teóricos de Frankfurt, si no que su fundamentación principal proviene de Marx y Grammsci, dado que su propuesta es mucho más práctica que teórica al reflexionar sobre el fenómeno educativo.

La Teoría Crítica en todas sus tres generaciones permea a la Pedagogía, pero principalmente las posiciones de Habermas desde la teoría general de la sociedad, la teoría general de la acción social y la teoría de la legitimidad, todas ellas determinadas por la acción comunicativa. La teoría de la acción comunicativa prevalece ya sobre la teoría del conocimiento; pues la relación sujeto-sujeto en la intersubjetividad del entendimiento posible, en el plano interpersonal como en el intrapsíquico, está mediada por la comunicación, el lenguaje, el diálogo. Esto es un cambio paradigmático importante, pues la tradición positivista dictaba que la teoría del conocimiento se centraba en la relación sujeto-objeto en el fenómeno. La teoría de la comunicación, pone en el debate la intersubjetividad en la praxis de un sujeto por naturaleza social, que se relaciona con otros sujetos en el actuar, el lenguaje y así le dan sentido al mundo subjetivo que comparten (Gaitán, 2014). 
Por ello, se infiere, que siendo el acto educativo un acto comunicativo, los postulados habermasianos encuentran en la praxis pedagógica un espacio de reflexión y estudio. La relación entre Pensamiento Crítico y Pedagogía Crítica es, por tanto, íntima, pues la segunda bebe de la primera. Los principios epistemológicos del pensamiento crítico, de cuestionamiento a los sistemas políticos totalitarios, los acuerdos sociales derivados de la comunicación, y la democracia como el sistema político más conveniente en la formación de un sujeto político, no le son ajenos a la Pedagogía Crítica, de hecho se "apropia" de esos principios, y se "presta" como episteme para transformar las prácticas y los discursos pedagógicos en el aula, esto a través de la construcción ético-política del profesor, en resistencia a una escuela como la reproductora de los discursos y prácticas sociales de dominación, con el propósito de formar un individuo crítico. El Pensamiento Crítico y la Pedagogía Crítica entran en sintonía y en acuerdos teóricos-conceptuales en torno a la idea de sociedad, libertad, igualdad, diálogo, solidaridad, emancipación, entre otros.

En términos generales, en Latinoamérica, se distinguen dos filos de investigación desde la Pedagogía Crítica: uno de carácter intrínseco a la educación, que estudia la construcción, de un lado, de un ideal de profesor crítico, ético y político, y de otro, la generación de políticas educativas críticas que permeen los currículos y las estrategias didácticas; y el otro filo de carácter sociopolítico que estudia los modelos reproduccionistas y comunicativos (Ortega, 2009).

Una de las aristas de discusión más importantes para la Pedagogía Crítica consiste en reflexionar en torno a la construcción social del conocimiento. En un acercamiento a este fenómeno social, McLaren (2005) establece algunos factores que determinan las interrelaciones entre la Pedagogía Crítica y la construcción social del conocimiento, ellas son: las formas de conocimiento, las clases sociales, la cultura, las formas culturales, la hegemonía y la ideología.

En cuanto a las formas de conocimiento, la pedagogía crítica cuestiona el cómo y por qué el conocimiento ha sido y es construido tal como se ha hecho en las sociedades occidentales; de igual forma, las clases de poder dominante imponen qué tipo de conocimientos y métodos son más legítimos que otros, es así como el conocimiento en matemáticas, ciencias naturales, y tecnológicas es más favorecido que el conocimiento obtenido de las ciencias sociales y humanas. McLaren diferencia tres formas de conocimiento: el conocimiento técnico (productivo) el cual puede ser medido y cuantificado; el conocimiento práctico referido al adquirido mediante el análisis de los fenómenos histórico-sociales; y el conocimiento emancipatorio aquel que intenta reconciliar e integrar la oposición dialéctica entre el técnico y el práctico en términos de un eslabón mediador. 
En la misma línea, McLaren (2005) se refiere a las clases desde las relaciones económicas, sociales y políticas que gobiernan la vida en un orden social dado impuesto. Las relaciones de clase evidencian las limitaciones, exclusiones como también los privilegios de la clase dominante. Además de la clase trabajadora, hoy día, se distinguen otras como los grupos raciales de negros, hispanos, asiáticos, árabes, entre otros; las mujeres, los discapacitados, los ancianos, y últimamente los inmigrantes y refugiados. El origen social del individuo, o sea su clase, es un determinante, inevitable e irreversible, de su destino escolar y profesional; debido a que tal origen social produce y reproduce, de entrada, el fenómeno de selección y de exclusión de las clases populares (Bourdieu y Passeron, 2001).

Otra relación de gran importancia corresponde a la cultura, la cual es esencial para la pedagogía crítica; ésta entendida como las prácticas, ideologías y valores a partir de los cuales diferentes grupos otorgan sentido a su mundo y sus condiciones de vida dadas, impuestas. Una posición crítica sobre la cultura ayuda a comprender quien tiene el poder y como es reproducido en la escuela y en el orden social preestablecido. La cultura es entendida, también, como una forma de producción en donde las clases dominantes y dominadas perciben sus desigualdades en relación al poder; es un campo de lucha en donde la producción, legitimación y circulación de conocimiento constituyen centros de discusión del conflicto.

Las formas culturales, entendida como los símbolos y prácticas sociales que expresan cultura, tales como la televisión, los videos, las películas, además de la forma de vestir, la música, la religión, la gastronomía, y claro, la educación, se han desarrollado a partir de los esfuerzos de los grupos por definir sus vidas en el entorno político y material. La escuela y la escolarización es también una forma cultural, como lo es el fútbol, por ejemplo. Al respecto McLaren (2005, p. 275), plantea:

Las formas culturales no existen separadas de los conjuntos de apuntalamientos relacionados con los medios de producción económica, la movilización del deseo, la construcción de valores sociales, las asimetrías entre poder y conocimiento, las configuraciones de ideologías y las relaciones de clase, raza y género.

La llamada cultura dominante ejerce control sobre los grupos subordinados a través de un fenómeno llamado la hegemonía, la cual hace alusión al mantenimiento de la dominación no solo por la fuerza, 
[...] si no por prácticas sociales consénsuales, formas sociales y estructuras sociales producidas en espacios específicos tales como la iglesia, el estado, la escuela, los medios masivos de comunicación, el sistema político y la familia.” (McLaren, 2005, p. 275).

De la mano de la hegemonía va la ideología, que permea toda la vida social y no hace alusión solo a la política, se refiere también a la producción y representación de ideas, valores y creencias, y la forma en que son comunicadas y experimentadas por las personas y los grupos, en pocas palabras, la ideología se refiere a la producción de sentidos y significados (McLaren, 2005). Las prácticas educativas, en muchos profesores, se han visto moldeadas por estas ideologías hasta el punto se asumirlas normales y habituales, son inconscientes de ellas, pues asumen que están socialmente construidas y obedecen a estructuras legítimamente cimentadas e inmodificables, es decir, se sienten impotentes ante ellas. En otras palabras, la ideología representa un imaginario, un vocabulario de estandarización, una gramática de designios sancionados, sostenidos y reproducidos por prácticas sociales particulares diseñadas para la dominación; es la ideología dominante.

Ante estos factores, la Pedagogía Crítica se enfrenta y está particularmente interesada en las interrelaciones entre el poder y el conocimiento. Los sistemas educativos capitalistas separan el conocimiento de los contextos de poder y lo tratan de forma pasiva y meramente técnica, el conocimiento es instrumental y por ello es dominado por las hegemonías de poder; en esto el profesor es un mero tecnócrata. Es medular en este debate el papel que juega el discurso sobre el cual se expresa el conocimiento.

Las prácticas discursivas, las reglas por las cuales se forman los mismos discursos, gobiernan lo que puede circular, lo que puede ser dicho, como también lo que debe ser callado, quién lo debe decir y quien debe solo escuchar (McLaren, 2005). La escuela y las instituciones gubernamentales son dominadas por este tipo de prácticas discursivas, las cuales están incorporadas en los tuétanos de los patrones de conducta social general donde los medios de comunicación capitalistas son amos y dueños del discurso que circula y que, sin duda, permea a la universidad y por ello, el profesor, se apropia de un discurso "pedagógico" prediseñado, impuesto, inconsciente y falaz que llega sin atisbos a los estudiantes.

La Pedagogía Crítica, y más el profesor radical, Giroux (2004), hoy en día es peligroso -es subversiva por su denuncia social- al menos ante los ojos de las comunidades religiosas y políticas conservadoras, ante los gobiernos serviles a los intereses del capital; pues su discurso se centra en la “... tarea de educar a los alumnos y alumnas para que se conviertan en agentes críticos que se 
cuestionan y discuten, de manera activa, la relación entre la teoría y la práctica, entre el análisis crítico y el sentido común, entre el aprendizaje y el cambio social” (McLaren y Kincheloe, 2008).

\section{Conclusiones}

El compromiso solidario con las formas de enseñanza y el aprendizaje de los agentes del acto educativo para la construcción de una conciencia social individual y colectiva; donde la participación, la comunicación, la humanización, la transformación, contextualización son elementos esenciales a tener en cuenta para un desarrollo humano.

Se reconoce a la Pedagogía Crítica como un cuerpo de pensamiento que ha de concebirse articulado a un proyecto de lucha pedagógica y de fortalecimiento político, que pretende provocar cambios en el sistema educativo, por ello aparece como una alternativa en coherencia con la Teoría Crítica, y en comunión con ella, aspira a la transformación social y a la renovación del discurso pedagógico de los profesores a través del análisis de la realidad desde la óptica del marxismo.

Desde este punto de vista, la Pedagogía Crítica es una concepción teórica en el marco de la sociología de la educación. Es medular para ella revelar los procesos de enseñanza y aprendizaje como escenarios de poder, políticos, históricos en contexto; cuestiona, tanto el conocimiento de sentido común cotidiano como producto y vivencia, como también, las funciones sociales del conocimiento científico; por ello, así mismo, el modelo y los sistemas educativos que se constituyen en prácticas culturales que determinan el futuro de los sujetos.

\section{Referencias}

Bourdieu, P. (1991). El sentido práctico. Madrid: Taurus.

Bourdieu, P. y Passeron, J. (2001). La reproducción: elementos para una teoría del sistema de enseñanza. Madrid: Editorial Popular.

Fonseca, R. (2011). Fundamentación teórica para una propuesta de pedagogía crítica. Tesis de Maestría en Educación. Bogotá: Universidad Nacional de Colombia.

Gaitán, C. (2014). Teoría Crítica de la sociedad y pedagogía Crítica. Revista Educación y Cultura No. 101, 35-41. Medellín: EAFIT.

Giroux, H. (2004). Teoría crítica y resistencia en educación. Buenos Aires: Siglo XXI editores.

López, G. (2010). Apuntes sobre la pedagogía crítica: su emergencia, desarrollo y rol en la posmodernidad. Volumen I. Cali: Universidad Santiago de Cali.

McLaren, P. (2005). La vida en las escuelas. Una introducción a la pedagogía critica en los fundamentos de la educación. México D.F.: siglo xxi editores, s.a. de c.v. 
McLaren, P. y Kincheloe, J. Eds. (2008). Pedagogía Crítica, de qué hablamos, dónde estamos. Barcelona: Editorial GRAÓ, de IRIF, S, L.

Morales, (2014). El pensamiento crítico en la teoría educativa contemporánea. Revista Actualidades Investigativas en Educación. Volumen 14, Número 2, mayo - agosto, pp. 1-23. San José: Universidad de Costa Rica.

Muñoz; C. (2016). El profesor universitario como intelectual transformador. Ponencia en Congreso Universidad 2016. La Habana: Universidad 2016.

Ortega, P. (2009). La Pedagogía Crítica: Reflexiones en torno a sus prácticas y sus desafíos. Bogotá: Universidad Pedagógica Nacional. 